\title{
Canine-specific PCR assay targeting cytochrome $b$ gene for the detection of dog meat adulteration in commercial frankfurters
}

\begin{abstract}
This report described a cytochrome b (cytb)-based polymerase chain reaction (PCR) assay for the detection of canine tissues in commercial frankfurters. Discriminating detection of canine derivatives in processed food products has important application in halal authentication as well as in health, religions, and fare trades. The assay based on a pair of canine-specific primers that targeted a $100 \mathrm{bp}$ region of canine mithochondrial-cytb gene which is present in multiple copies and highly conserved within the same species. The specificity of the assay was tested against dog and eight most common animal meat species as well as five plant species commonly found in frankfurter formulation. The stability and specificity of the assay were verified under different thermal processing conditions under pure and complex matrices. Three commercial brands of chicken and beef frankfurters were tested in triplicate, and specific PCR products were obtained only from deliberately contaminated formulations. The detection limit of the assay was $0.1 \%(0.02 \mathrm{ng}$ DNA) of canine meat spiked with other meats in a typical frankfurter formulation. Shorter amplicon length, superior stability, and higher sensitivity of the assay suggested its potential application in the screening of canine-origin biomaterials in processed food products.
\end{abstract}

Keyword: Canine-origin biomaterials; Commercial frankfurters; Halal authentication 